\title{
The Volumetric Measurement of Gastric Emptying and Gastric Secretion by a Radioisotope Method
}

\author{
RONALD A. HINDER, MB, BCh (Rand), FRCS (Ed), FRCS (Eng), BERNARD K.P. \\ HORN, PhD, and CEDRIC G. BREMNER, MCh (Rand), FRCS (Ed), FRCS (Eng)
}

\begin{abstract}
Most methods of measurement of gastric emptying rely on the serial estimation of intragastric volume and do not separately account for the volume of fluid which has been added to the meal by gastric secretion, duodenal reflux, or swallowed saliva. The volume emptied is therefore underestimated. A method of measuring gastric emptying using [ $\left.{ }^{225} I\right] R I H S A$ and the Volemetron is presented. The volume of fluid added to the meal is taken into consideration in this method, giving a more accurate reflection of gastric emptying. Using this method in the dog, emptying was found to be linear rather than exponential.
\end{abstract}

We have previously reported a method of measurement of intragastric volume using the Volémetron and $\left[{ }^{125} \mathrm{I}\right]$ RIHSA (radioiodinated human serum albu$\min )(1)$. The Volémetron is an apparatus, using an isotope dilutional technique, normally used for the measurement of blood volumes. The apparatus measures the radioactivity of a dose of $\left[{ }^{125}\right.$ I] RIHSA, which is then injected into a volume of fluid to be measured. Specimens $(2 \mathrm{ml})$ of the volume of fluid are taken before (premix) and after (postmix) the injection of $\left[{ }^{125} I\right]$ RIHSA and have their radioactivity measured in the Volémetron. With the information of the change of isotope concentration afforded by the injection of a known amount of [ ${ }^{125}$ I]RIHSA, the apparatus is able to automatically compute and display this volume. Repeated estimations of the same

From the Department of Surgery, University of the Witwatersrand, Medical School, Esselen Street, Johannesburg 2001, Republic of South Africa.

Dr. Horn is now Assistant Professor at the Artificial Intelligence Laboratory, Massachusetts Institute of Technology, Cambridge, Massachusetts 02139.

Supported by South African Medical Research Council grant M14/71/51.

Address for reprint requests: Dr. R. A. Hinder, Department of Surgery, University of the Witwatersrand, Medical School, Esselen Street, Johannesburg 2001, Republic of South Africa. fluid volume may be carried out, each time adding a new dose of [ ${ }^{125}$ I]RIHSA.

When this method is applied to the measurement of gastric emptying it has the same disadvantages of other dilution methods in that it relies on serial estimations of intragastric volumes and does not account separately for the volume added by simultaneous gastric secretion, duodenal reflux, or swallowed saliva. These methods therefore give only a rough estimate of gastric emptying, and the volume of gastric emptying deduced is therefore underestimated.

An elaboration of our method now measures intragastric dilution due to these other components. We are therefore able to calculate more accurately actual gastric emptying.

\section{MATERIALS AND METHODS}

Seven adult mongrel dogs of both sexes weighing approximately $15 \mathrm{~kg}$ were trained to accept an F18 doublelumen orogastric tube of the Salem type (Sheridan Corporation, New York) using a mouth gag. No sedation was used. When the dogs accepted the tube without agitation, they were used for testing. The dogs were starved of food but not water for $24 \mathrm{hr}$ prior to testing. Only one test per day was carried out on any particular dog. A milk feed 


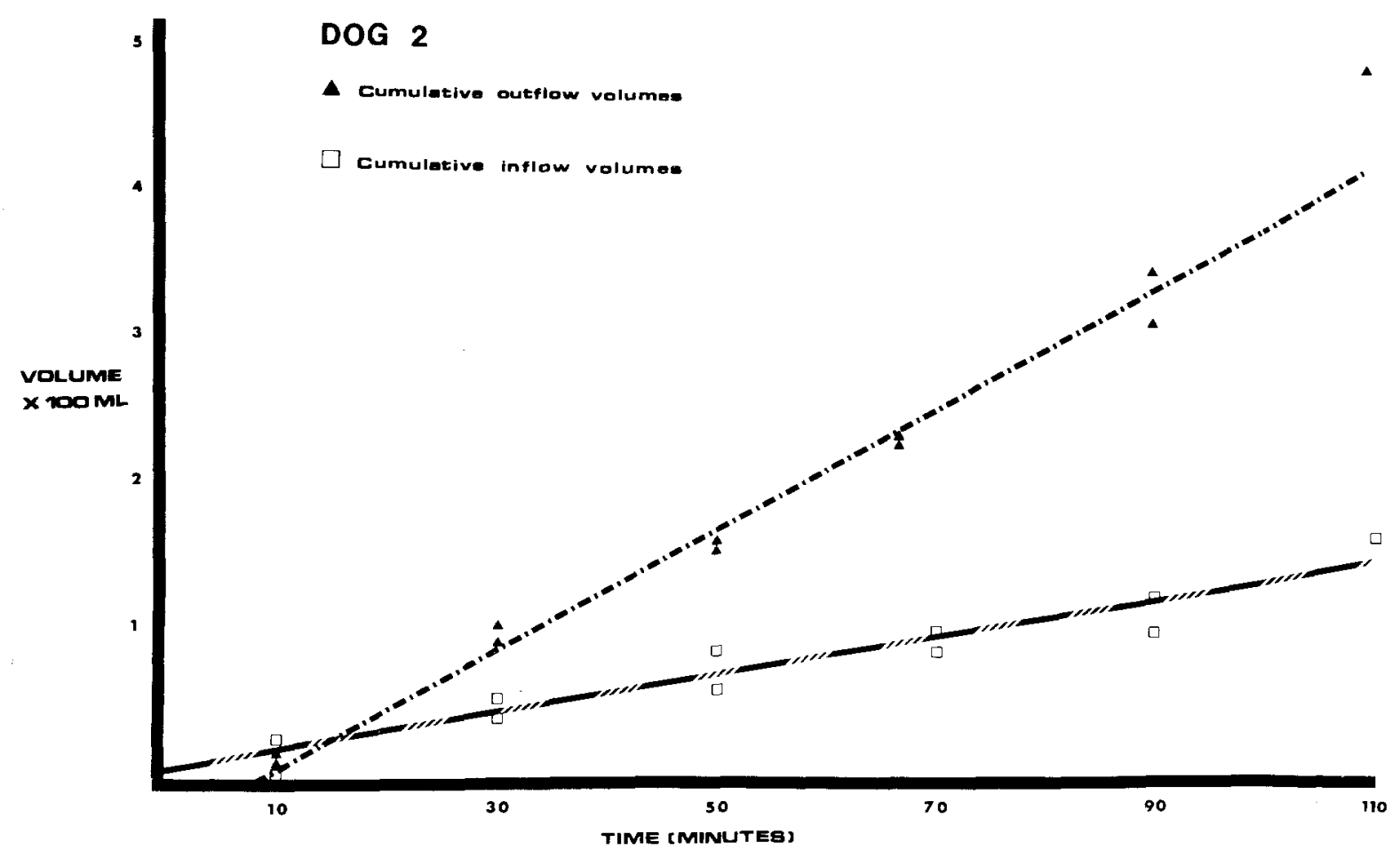

Fig 1. Inflow and outflow volumes plotted against time, with their respective computed regression lines following two separated milk meals.

was made up by diluting $110 \mathrm{ml}$ of Carnation milk with $190 \mathrm{ml}$ of water.

The tube was passed and the stomach washed out with $200 \mathrm{ml}$ of saline via the broader lumen of the tube using a $100-\mathrm{ml}$ bulb syringe. If food was present in the return or the return was below $160 \mathrm{ml}$, the test was discontinued. This occurred infrequently. A volume of $20 \mathrm{ml} / \mathrm{kg}$ body weight of the reconstituted Carnation milk, plus a further $12 \mathrm{ml}$ of the milk was used as the test meal. A $0.6-\mu \mathrm{Ci}$ dose $(0.35 \mathrm{ml})$ of [ $\left.{ }^{125} \mathrm{I}\right]$ RIHSA was added to the meal. The meal was rapidly passed into the stomach, via the broader lumen of the tube, using the bulb syringe. Adequate mixing of the meal in the stomach was obtained over about 1 min by rapid to-and-fro aspiration of the meal with the bulb syringe. A 12-ml specimen (specimen 1) of the meal was aspirated and divided into $10-\mathrm{ml}$ and $2-\mathrm{ml}$ portions to be used later. The tube and gag were then removed. The dogs were kept free of restraint and on all fours at all times. The tube was again passed $10 \mathrm{~min}$ later and the meal well mixed for approximately $1 \mathrm{~min}$ by to-and-fro aspiration with the bulb syringe, via the broader lumen of the tube. A 2-ml premix specimen of the stomach contents was then taken for measurement in the Volémetron. Then a $0.35-\mathrm{ml}$ dose of $\left[{ }^{125} \mathrm{I}\right] \mathrm{RIHSA}$, the radioactive count of which had been previously measured and stored by the Volémetron, was injected into the meal via the fine bore lumen of the tube. This was washed through with $10 \mathrm{ml}$ of specimen 1. Following further adequate intragastric mixing as above, a $12-\mathrm{ml}$ postmix specimen of gastric contents was taken. A $10-\mathrm{ml}$ portion of this specimen was kept to wash through the dose of $\left[{ }^{125} I\right]$ RIHSA during the next estimation of intragastric volume $20 \mathrm{~min}$ later, and the remaining $2 \mathrm{ml} \mathrm{kept}$ for measurement in the Volémetron. The tube and gag were again removed. The premix and postmix specimens then had their radioactive counts measured by the Volémetron. Now with the knowledge of the change of isotope concentration produced by the injection of a measured amount of isotope, the Volémetron was able to automatically compute and display the intragastric volume. Then $10 \mathrm{ml}$ was subtracted from this reading to allow for the volume of fluid added to wash through the $\left[{ }^{125} I\right]$ RIHSA. Every $20 \mathrm{~min}$ the Volémetron was used to calculate the intragastric volume by applying the same procedure as above: The tube was passed, and a 2-ml premix specimen obtained; a new

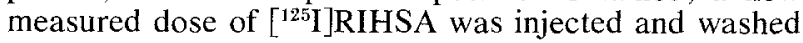
through with $10 \mathrm{ml}$ of the postmix specimen of the previous estimation; and finally a new $12-\mathrm{ml}$ postmix specimen was taken, and the tube removed. The test was discontinued when the intragastric volume approached approximately $50 \mathrm{ml}$, when the stomach contents were completely aspirated and measured in a measuring cylinder as a check. The remaining $2 \mathrm{ml}$ of specimen 1 and all the subsequent premix and postmix samples each had 
Table 1. Regression Constants of Inflow Volumes (Linear and Log) Against Time, Following Milk Meals, with Relative Correlation Coefficients

\begin{tabular}{|c|c|c|c|c|c|}
\hline & \multirow{2}{*}{$\begin{array}{c}\text { Number of } \\
\text { observations }\end{array}$} & \multicolumn{2}{|c|}{ Regression constants } & \multicolumn{2}{|c|}{ Correlation coefficients } \\
\hline & & Linear & $\log$ & Linear & $\log$ \\
\hline $\operatorname{Dog} 1$ & 4 & 0.53 & 0.004 & 0.9627 & 0.9816 \\
\hline $\operatorname{Dog} 1$ & 4 & 1.11 & 0.006 & 0.9762 & 0.9465 \\
\hline $\operatorname{Dog} 1$ & 4 & 0.86 & 0.012 & 0.9240 & 0.9469 \\
\hline $\operatorname{Dog} 2$ & 6 & 1.38 & 0.008 & 0.9885 & 0.9652 \\
\hline $\operatorname{Dog} 2$ & 5 & 1.16 & 0.006 & 0.9819 & 0.9935 \\
\hline Dog 3 & 7 & 0.64 & 0.023 & 0.9701 & 0.9341 \\
\hline Dog 3 & 6 & 1.01 & 0.016 & 0.9731 & 0.9599 \\
\hline $\operatorname{Dog} 4$ & 6 & 0.27 & 0.003 & 0.9299 & 0.8955 \\
\hline $\operatorname{Dog} 4$ & 6 & 1.82 & 0.007 & 0.8837 & 0.8274 \\
\hline Dog 5 & 3 & 1.40 & 0.015 & 0.9981 & 0.9722 \\
\hline $\operatorname{Dog} 5$ & 4 & 0.96 & 0.007 & 0.9960 & 0.9731 \\
\hline $\operatorname{Dog} 6$ & 5 & 0.72 & 0.016 & 0.9617 & 0.9721 \\
\hline Dog 6 & 5 & 1.68 & 0.016 & 0.9834 & 0.9175 \\
\hline $\operatorname{Dog} 7$ & 6 & 0.24 & 0.017 & 0.7422 & 0.6151 \\
\hline $\operatorname{Dog} 7$ & 6 & 0.70 & 0.028 & 0.9730 & 0.8508 \\
\hline
\end{tabular}

their radioactive counts measured over 2 min in a Packard Auto Gamma Spectrometer. The results were compared as follows for each 20 -min period.

\section{Mathematical Model}

Fluid Exchanges. The volume of fluid in the stomach at the start of a period between measures of intragastric volume contains $\left[{ }^{125} \mathrm{I}\right] \mathrm{RIHSA}$ at a known concentration. This information is obtained by measuring the radioactive counts of specimen 1 for the first 10 -min period and of each of the postmix specimens for the subsequent 20 -min periods. There are two kinds of fluid exchanges between the stomach and its environment which may change this concentration.

1. Fluid containing little or no $\left[{ }^{125} I\right]$ RIHSA such as gastric secretion, gastric absorption, saliva inflow, and fluid returned across the pylorus. Most of the flows are into the lumen. Let the total volume of "inflow" of fluid not containing [ $\left.{ }^{125} \mathrm{I}\right]$ RIHSA for a 20 -min period be A.

2. Fluid containing $\left[{ }^{125} \mathrm{I}\right] \mathrm{RIHSA}$, mostly being outflow across the pylorus. Let the total volume of "outflow" of fluid containing [ ${ }^{125}$ I] RIHSA for a $20-$ min period be B.

At the start of the time interval we measure the volume and concentration $V_{0}$ and $C_{0}$. This is repeated at the end of the time interval, to obtain $V_{1}$ and $C_{1} . C_{1}$ is obtained by measuring the radioactive counts of the premix specimens. Under the assumption of perfect mixing and constant flow rates, it can be shown that the total inflow $A$ and the total outflow $\mathbf{B}$ over the time interval are given by:

$$
A=\left(V_{0}-V_{1}\right) \frac{\log \left(C_{1} / C_{0}\right)}{\log \left(V_{1} / V_{0}\right)}
$$

$$
B=A+\left(V_{0}-V_{1}\right)
$$

(See appendix for full derivation.)

If mixing is not perfect and the flow rates are not constant, one cannot arrive at a unique value for the total inflow $A$, but it can be shown that it has to lie in the range given by the following:

$$
V_{1}\left(1-\frac{C_{1}}{C_{0}}\right)<A<V_{0}\left(\frac{C_{0}}{C_{1}}-1\right)
$$

The total outflow B can once again be calculated using equation (2). In practice the in- and outflows are indeed not perfectly steady and probably pulsative, but do average out over a sufficiently long time interval. Under these circumstances the best estimate for $\mathrm{A}$ and $\mathrm{B}$ are given by equations (1) and (2). The extremes in equation (3) are only obtained in the unlikely situation of there being no overlap between a single period of inflow and a single period of outflow.

\section{RESULTS}

Figure 1 represents a typical example of the inflow and outflow patterns obtained in two milk tests on the same normal $\operatorname{dog}(\operatorname{dog} 2)$. The regression constants of volume against time are shown. Tables 1 and 2 show the regression constants of milk meals in the 7 dogs of volume and log of volume of inflow and outflow against time. The relative correlation coefficients show that a more satisfactory correlation with a straight line occurs when the volume as opposed to the log of volume is plotted against time. 
Table 2. Regression Constants of Outflow Volumes (Linear and Log) against Time, Following Milk Meals, with Relative Correlation Coefficients

\begin{tabular}{|c|c|c|c|c|c|}
\hline & \multirow[b]{2}{*}{$\begin{array}{c}\text { Number of } \\
\text { Observations }\end{array}$} & \multicolumn{2}{|c|}{ Regression constants } & \multicolumn{2}{|c|}{ Correlation coefficients } \\
\hline & & Linear & $\log$ & Linear & $\log$ \\
\hline Dog 1 & 4 & 3.33 & 0.011 & 0.9869 & 0.9376 \\
\hline Dog 1 & 4 & 4.43 & 0.019 & 0.9939 & 0.9665 \\
\hline $\operatorname{Dog} 1$ & 4 & 2.63 & 0.007 & 0.9896 & 0.9996 \\
\hline $\operatorname{Dog} 2$ & 6 & 4.40 & 0.014 & 0.9901 & 0.8970 \\
\hline $\operatorname{Dog} 2$ & 5 & 3.74 & 0.015 & 0.9981 & 0.9325 \\
\hline Dog 3 & 7 & 2.24 & 0.010 & 0.9856 & 0.9243 \\
\hline $\operatorname{Dog} 3$ & 6 & 2.49 & 0.016 & 0.9667 & 0.8702 \\
\hline $\operatorname{Dog} 4$ & 6 & 1.97 & 0.009 & 0.9615 & 0.9661 \\
\hline $\operatorname{Dog} 4$ & 6 & 4.34 & 0.011 & 0.9779 & 0.8779 \\
\hline $\operatorname{Dog} 5$ & 3 & 3.70 & 0.014 & 0.9880 & 0.9997 \\
\hline $\operatorname{Dog} 5$ & 4 & 2.63 & 0.008 & 0.9879 & 0.9556 \\
\hline $\operatorname{Dog} 6$ & 5 & 4.13 & 0.010 & 0.9823 & 0.9332 \\
\hline Dog 6 & 5 & 4.22 & 0.010 & 0.9704 & 0.9145 \\
\hline $\operatorname{Dog} 7$ & 6 & 2.94 & 0.027 & 0.9882 & 0.7310 \\
\hline $\operatorname{Dog} 7$ & 6 & 2.60 & 0.028 & 0.9942 & 0.7593 \\
\hline
\end{tabular}

\section{DISCUSSION}

All previous methods of measuring gastric emptying, in which gastric volume was used as a reflection of emptying, have ignored the dilution of the gastric juice by gastric secretion, duodenal reflux, and swallowed saliva. This error is diminished by our method.

A method of measuring the volume of fluid added to the gastric contents over a period of time is also offered. This volume is largely gastric secretion. Hunt (2) has previously reported a method of measuring the volume of fluid added to a meal by the parietal and nonparietal component of the stomach. His method relies on measuring the amount of hydrogen and chloride ion in the gastric content, and then, assuming a constant concentration of these ions in gastric secretions, an estimate is made of the volume secreted. If duodenal reflux can be measured and excluded (3), we feel that our method would be at least as accurate in estimating the volume of gastric secretion as that of Hunt. If there were an accurate way of measuring the volume of gastric secretion, this result could be subtracted from the total inflow, and if swallowed saliva was excluded, the result would then indicate the volume of duodenal reflux.

Similar methods of measurement of inflow and outflow using nonisotopic markers have been previously reported $(4,5)$. Hunt felt that these refinements "complicated the already tedious arithme- tic." But our method only involves the solution of two rather simple equations, and gives greater accuracy of measurement of gastric emptying. A further advantage is that this method uses an isotopic marker and thus allows for the use of a non-water-clear meal containing carbohydrate, protein, and fat, which cannot be used in spectrophotometric methods.

The formulae presented by us may also be used to calculate inflow and outflow from the basic data obtained in dye dilution tests of intragastric volume such as those of George (6).

In vitro testing of our method simulating the inflow and outflow conditions as seen in vivo has shown this method to be very accurate. In our method errors in estimation may occur if there is reflux of isotope-containing fluid from the duodenum. This volume would not be fully accounted for in the inflow volume. Another possible error in estimation could be produced by peptic digestion of the albumin and subsequent loss of ${ }^{125} \mathrm{I}$ from the meal. Also fluid absorption from the stomach may result in concentration of the isotope, or alternatively secretion of [ ${ }^{125}$ I] RIHSA into the stomach after absorption in the small bowel may occur. Change of isotope concentration due to these factors has been studied in a milk meal in the pyloric-ligated stomach and in a dog Heidenhain pouch over the same time period as the test period. Samples $(2 \mathrm{ml})$ of the meal in the pyloric-ligated stomach of a dog under barbiturate gen- 
eral anesthesia were taken at 20 -min periods over a time period of $100 \mathrm{~min}$. The isotope concentration remained within $3 \%$ of the mean. Some fall in isotope concentration was observed, probably due to the dilutional effect of gastric secretion. The results were found not to change when a large dose of [ ${ }^{125}$ I] RIHSA was injected into the small bowel during the experiment. The same result was obtained in the Heidenhain-pouch experiment.

With our method it has been found that a better correlation of experimental points to the lines of regression for outflow occurs when plotting the volumes in a linear fashion as opposed to a log plot (Table 2). Applying the Wilcoxon rank test to these data, it was found that they were significantly different $(P<0.05)$. Thus using this method of measurement of gastric emptying the "classical" exponential pattern of emptying (4) was not shown. It may be that the volume of the original meal empties in an exponential fashion, but our data suggests that this is not so when studying the emptying of the whole gastric contents. It has been previously shown that the volume of gastric secretion in the dog is 60-150. $\mathrm{ml} / \mathrm{hr}(7)$. We have obtained similar results. This further supports the accuracy of our method.

\section{APPENDIX-MATHEMATICAL MODEL}

Suppose a volume $V(t)$ of fluid contains an amount $Q(t)$ of some tracer substance. Define the concentration $C(t)=Q(t) / V(t)$. All these quantities may vary with time due to the effect of tracer-free fluid being added and fluid containing tracer being removed. Suppose next that over an interval of time $T$ a total volume $A$ flows in and volume $B$ flows out. Then the total change in volume is simple $(A-B)$. Unfortunately the change in concentration is not so easily calculated, since it depends on the time history of the relative rates of in- and outflow as well as the degree of mixing. To understand this, it is best to look at extreme cases first and then try and obtain a good average estimate for more realistic situations.

Let $V_{0}, C_{0}$ be the initial volume and concentration, and $V_{1}, C_{1}$ the volume and concentration at the end of the time interval $T$. Then clearly $\left(V_{1}-V_{0}\right)=(A-B)$. If there is no inflow, the concentration of the tracer substance will not change and so $C_{1}=C_{0}$. If on the other hand there is no outflow, the total quantity of tracer substance remains unchanged and so $C_{1}\left(V_{0}+A\right)=C_{0} V_{0}$. These formulae can now be used to calculate the final concentration given alternating periods of in- and outflow.
Given fixed amounts of inflow $A$ and outflow $B$, what distribution in time of flows will give rise to the lowest possible final concentration? If all the outflow precedes all the inflow, the largest amount of tracer substance is lost and hence the lowest final concentration will obtain.

$$
\frac{C_{1}^{\prime}}{C_{0}}=\frac{V_{0}-B}{V_{0}+(A-B)}=\frac{V_{1}-A}{V_{1}}
$$

The highest final concentration, on the other hand, is obtained if one reverses the above procedure and allows all of the inflow to occur before all of the outflow.

In that case:

$$
\frac{C_{1}^{\prime \prime}}{C_{0}}=\frac{V_{0}}{V_{0}+A}=\frac{V_{1}-(A+B)}{V_{1}+B}
$$

If there are several alternating periods of in- and outflow, one can apply the above formulae to each such interval. The final concentration will always fall between the two extremes in equations (4) and (5). In fact, the more the periods of inflow are intermingled with the periods of outflow, the further the final concentration will lie from both extremes, and the closer it will approach some intermediate value corresponding to a steady rate of inflow, $a=A / T$, and a steady rate of outflow, $b=B / T$. This value will be approached even more closely if the periods of in- and outflow overlap. If one does not have a detailed time history of in- and outflows, one can obtain a good estimate of final concentration by assuming steady in- and outflow. To obtain this estimate one has to solve a differential equation.

The rate at which tracer substance is lost is simply the product of its concentration and the rate of outflow.

$$
\frac{d Q}{\mathrm{dt}}=\frac{d}{d t}(V C)=-C b
$$

Now invoke the chain rule for differentiation $\left(\frac{d Q}{d t}=\right.$

$$
\left.\frac{d Q}{d V}=\frac{d V /}{d t}\right): \quad \frac{d}{d V} \text { (VC) } \frac{d V}{d t}=-C b
$$

The rate of change of volume, $d V / d t=(a-b)$. Next, differentiate the product:

$$
\left(C+V \frac{d C}{d V}\right)(a-b)=-C b
$$


Rearranging terms one gets the differential equation:

$$
\frac{d C}{d V}=\frac{a}{b-a} \frac{C}{V}=\frac{A}{B-A}\left(\frac{C}{V}\right)
$$

This equation is separable.

Integrating this equation over the time interval $T$ one gets:

$$
\begin{gathered}
\log \left(\frac{C_{1}}{C_{0}}\right)=\frac{A}{B-A} \log =\left(\frac{V_{1}}{V_{0}}\right) \\
\frac{C_{1}^{\prime \prime \prime}}{C_{0}}=\left(\frac{V_{1}}{V_{0}}\right) \frac{A}{B-A}
\end{gathered}
$$

The above solution will apply except for the degenerate case when $V_{1}=V_{0}$. Solving the differential equation separately for this special case leads to:

$$
\begin{gathered}
\log _{e}\left(\frac{C_{1}}{C_{0}}\right)=-\frac{A}{V_{0}} \\
\frac{C_{1}^{\prime \prime \prime}}{C_{0}}=e^{-\left(A / V_{0}\right)}
\end{gathered}
$$

So far the implicit assumption has been that the fluid continues to be mixed well during the time interval $T$ (the experimental procedure ensures that it is well mixed at the beginning and end of the time interval). Again, first consider the extreme caseno mixing. In this case the inflow remains near the point of entry, is not mixed in, and so does not contribute to the outflow. This is equivalent to allowing all the outflow to precede all of the inflow and hence gives a low final concentration. This will however still be within the limits previously established in equations (4) and (5). For more realistic mixing rates, the final concentration approaches the estimate in equation (11).

Thus one has both a range for the final concentration given arbitrary distributions in time of in- and outflows as well as mixing rates and a best estimate based on the assumption that in- and outflow rates are steady and mixing is perfect. One can now turn these formulae around to calculate total inflow $A$, given measured final concentrations. The lowest value for $A$ will be obtained if one uses equation (4):

$$
A^{\prime}=V_{1}\left(1-\frac{C_{1}}{C_{0}}\right)
$$

The highest value for $A$, on the other hand, comes from the use of equation (5):

$$
A^{\prime \prime}=V_{0}\left(\frac{C_{0}}{C_{1}}-1\right)
$$

Finally, the best estimate, obtained from equation (10) is:

$$
A^{\prime \prime \prime}=\left(\dot{V}_{0}-V_{1}\right) \frac{\log \left(C_{1} / C_{0}\right)}{\log \left(V_{1} / V_{0}\right)}
$$

Except for the degenerate case when $V_{1}=V_{0}$, when:

$$
A^{\prime \prime \prime}=V_{0} \log _{e}\left(C_{0} / C_{1}\right)
$$

One can get an idea of how large is the range of uncertainty for possible flow rates that yield a given final concentration by dividing $A^{\prime \prime}$ by $A^{\prime}$.

$$
\frac{A^{\prime \prime}}{A^{\prime}}=\frac{V_{0} C_{0}}{V_{1} C_{1}}=\frac{Q_{0}}{Q_{1}}
$$

In any case it should be clear that even a small uncertainty in the determination of total in- and outflows has to be preferrable to pretending that there are no inflows.

\section{ACKNOWLEDGMENT}

We wish to thank Professor D. J. du Plessis for his criticism and facilities given in his department and alsö Mr. P. R. Tuffin and Dr. J. Ridley for statistical and mathematical aid.

All experiments were performed in the Michael and Janie Miller Experimental Laboratory.

\section{REFERENCES}

1. Hinder RA, Bremner CG: A radioisotope method of measuring intragastric volumes using the Volémetron. Gut 14:113115,1973

2. Hunt JN: Secretory pattern of the stomach. J Physiol 113:169-184, 1951

3. Gardham JRC, Hassan MA, Hobsley. M: The recognition and measurement of pyloric reflux in aspirated gastric juice. Br J Surg 55:865, 1968

4. Hunt JN: Gastric emptying and secretion in man. Phys Rev 39:491-533, 1959

5. Schoen AM, Knoefel PK: The measurement of human gastric function. J Lab Clin Med 32:345-346, 1947

6. George JD: New clinical method for measuring the rate of gastric emptying: The double sampling test meal. Gut 9:237-242, 1968

7. Weisbrodt NW, Wiley JN, Overholt BF, Bass P: A relation between gastroduodenal muscle contractions and gastric emptying. Gut 10:543-548, 1969 